\title{
Adherence to, and outcomes of, a galactomannan screening protocol in high-risk hematology patients
}

S. Harricharan MPharm, ${ }^{*}$ K. Biederman $\mathrm{BScPhm}^{\dagger}{ }^{\dagger}$ A.M. Bombassaro BScPhm PharmD, ${ }^{\text {t† }}$ A. Lazo-Langner MD MSc, ${ }^{\S} \|$ S. Elsayed $\mathrm{MD}_{,}{ }^{* * *}$ A. Fulford $\mathrm{MScN}_{,}{ }^{+\dagger}$ J.A. Delport MMed MBChB, ${ }^{* * \neq \neq}$ and A. Xenocostas $M D^{\S}$

\section{ABSTRACT}

Background A twice-weekly galactomannan (GM) screening protocol was implemented in high-risk hematology inpatients. Study objectives were to determine adherence to the protocol, use of selected resources, and patient outcomes.

Methods This retrospective cohort study compared outcomes of interest before and after implementation of GM screening. Adults undergoing matched related allogeneic hematopoietic stem-cell transplantation or induction chemotherapy for acute leukemia were eligible. Patients could be enrolled more than once and were evaluated as episodes. Adherence to the GM protocol was assessed in post-implementation episodes. Use of broad-spectrum antifungals (BSAFs), consultations (infectious diseases, respirology), and diagnostic procedures (computed tomography imaging, bronchoalveolar lavage) were compared between phases, as were the patient outcomes of all-cause mortality and clinical success (alive and not taking a BSAF).

Results Of 182 episodes consecutively screened, 70 per phase were enrolled. Clinical characteristics and duration of assessment were similar for the phases. Full or partial adherence to the protocol was observed in 61 postimplementation episodes (87\%), with full adherence in 40 episodes (57\%). More episodes in the pre-implementation phase than in the post-implementation phase involved receipt of BSAFs, consultations, and diagnostics $(27 \%$ vs. $7 \%$, $p=0.02 ; 46 \%$ vs. $26 \%, p=0.014$; and $46 \%$ vs. $31 \%, p=0.083$ respectively). Although mortality was similar in the two phases, clinical success at the final assessment was observed in fewer pre-implementation than post-implementation episodes $(79 \%$ vs. $98 \%, p<0.001)$.

Conclusions Implementation of a GM screening protocol was feasible and associated with significantly fewer episodes involving receipt of BSAFS and consultations, and with significantly more episodes showing clinical success.

Key Words Adherence, antifungals, consultations, diagnostics, galactomannan, hematology, mortality, screening Curr Oncol. 2018 April;25(2):e139-e145 www.current-oncology.com

\section{INTRODUCTION}

Invasive fungal disease (IFD) is a significant cause of morbidity and mortality in patients undergoing allogeneic hematopoietic stem-cell transplantation (allo-HSCT) and in those receiving chemotherapy for hematologic malignancies ${ }^{1}$. Empiric antifungal therapy to treat suspected IFD has been the standard of care for neutropenic patients who have persistent fever despite treatment with broadspectrum antibacterials. However, the appropriateness of using fever as the only indicator for initiating antifungals has been challenged, because that approach might lead to overtreatment ${ }^{2-6}$.

Development of noninvasive diagnostic markers, such as galactomannan (GM), $\beta$-D-glucan, and polymerase chain reaction methods, have led to interest in replacing empiric 
therapy with a pre-emptive approach for persistently febrile neutropenic patients ${ }^{7-9}$. The same approach might be an effective way to target antifungal use, thereby minimizing the risk of drug-related complications, resistance, and costs $^{1}$. A meta-analysis comparing empiric and pre-emptive antifungal strategies in patients with hematologic malignancy who have a high risk of febrile neutropenia showed that the pre-emptive strategies were associated with lesser antifungal exposure without an increase in IFD-related or overall mortality ${ }^{10}$.

The GM enzyme-linked immunosorbent assay is a noninvasive diagnostic test that detects circulating GM, a major constituent of the Aspergillus polysaccharide cell wall that is released during growth ${ }^{11}$. Detection of GM in various body fluids is included as a diagnostic criterion for probable invasive aspergillosis by the European Organisation for Research and Treatment of Cancer/Invasive Fungal Infections Cooperative Group and the National Institute of Allergy and Infectious Diseases Mycoses Study Group ${ }^{12}$. Prospective studies that have incorporated the GM assay as the sole non-culture-based marker into a diagnosticdriven pre-emptive strategy in patients with hematologic malignancy have demonstrated decreased use of empiric antifungals without an increase in mortality ${ }^{1,9,13}$.

In 2011 at our centre, fluconazole prophylaxis became a standard of care for inpatients undergoing either allo-HSCT or induction chemotherapy for acute leukemia who are at high risk of IFD. In December 2013, a twice-weekly GM screening protocol was implemented in the same population. The primary objective of the present study was to determine adherence to the GM screening protocol. Secondary objectives were to compare patient outcomes and the use of broad-spectrum antifungals (BSAFs) and consultative and diagnostic services before and after implementation of GM screening.

\section{METHODS}

\section{Design and Study Population}

This retrospective cohort study enrolled adult hematology patients at high-risk of IFD admitted to the Hematology Service of the London Health Sciences Centre, a tertiary care centre in London, Ontario. The Western University Office of Research Ethics reviewed and exempted the submission from the need for ethics approval because the study fulfilled the criteria of a quality improvement initiative.

Patients were eligible for inclusion if they were 18 years of age or older and were undergoing matched related allo-HScT or induction chemotherapy for acute myeloid leukemia (AML) or acute lymphoid leukemia (ALL). Patients could be included in the study more than once, and therefore the assessment considered patient episodes. An "episode" referred to a patient admission beginning on day 0 of stem-cell infusion for allo-HSCT or on day 1 of induction chemotherapy for AML or ALL and continuing until either white cell count recovery (absolute neutrophil count $0.5 \times 10^{9} / \mathrm{L}$ or greater, or total peripheral leucocyte count $1 \times 10^{9} / \mathrm{L}$ or greater if a neutrophil count was not available), discharge, death, or the start of the next chemotherapy cycle, whichever occurred first. Exclusion criteria were death occurring before the first protocol day of GM screening, receipt of at least 3 consecutive days of BSAF during the week before the start of the episode, or the availability of a GM result from an external centre during the episode.

Episodes were identified from computerized pharmacy records for specific chemotherapy protocols defined $a$ priori and prescribed during the study periods. Episodes in the phase before implementation of the GM protocol were consecutively screened for inclusion (September 2013 backward) until a convenience sample, based on available study resources, of 70 episodes was reached. Similarly, 70 episodes were enrolled for the phase after implementation of the GM protocol (February 2014 forward). The 4-month interruption between the "before" and "after" phases allowed for the implementation of GM screening in December 2013 and for a period of clinician adjustment to the new protocol through to the end of January 2014. Care decisions with respect to a patient's drug therapy, consultations, and diagnostics were at the discretion of clinicians in both study phases.

\section{Data Collection}

An electronic data collection tool was developed and piloted over a 4-week period. Operational definitions were established a priori, and data collection was performed by a single investigator. A quality audit of the data collected for $10 \%$ of the episodes in both phases was performed independently by a co-investigator not involved in the original data collection.

These data were captured from electronic and paper medical records: age, sex, hematologic diagnosis, neutropenia (defined as an absolute neutrophil count of $<0.5 \times 10^{9} / \mathrm{L}$ or a total peripheral leucocyte count of $<1 \times 10^{9} / \mathrm{L}$ if a neutrophil count was not available), targeted medications (chemotherapy, antifungals, broad-spectrum antibiotics), consultations (infectious diseases, respirology), diagnostic procedures (GM screens, computed tomography imaging, bronchoalveolar lavage), hospital discharge, and death. The indication for antifungals was determined from a review of the health record during the 3-day period before and after the prescription. Occurrence of a complication attributed to a BSAF was identified during the 3-day period before and after antifungal discontinuation, switch to an alternative agent, or change in dose.

\section{Study Outcomes}

The primary outcome was the proportion of episodes with adherence (full and partial) to the GM screening protocol in the post-intervention phase. "Full adherence" referred to the performance of all recommended twice-weekly screens during the episode or until 2 consecutive serum samples with a GM index of 0.5 or greater were attained. "Nonadherence" referred to no GM screens being performed. "Partial adherence" referred to episodes having other than full adherence or nonadherence.

Secondary outcomes included the proportion of episodes in the pre- and post-implementation phases involving receipt of a BSAF (amphotericin B, voriconazole, or caspofungin) for at least 3 consecutive days; consultations (infectious diseases, respirology) and diagnostic procedures (computed tomography imaging, bronchoalveolar lavage) 
for infection-related workup; all-cause mortality and clinical success (defined as alive and not taking a BSAF). The latter two outcomes were assessed at 6 weeks after the end of the episode or at day -1 before subsequent chemotherapy.

\section{Data Analysis}

Baseline characteristics are described using measures of central tendency. The primary outcome of adherence was determined for post-implementation episodes overall and for the underlying diagnosis of allo-HSCT, AML, or ALL. Adherence in the post-implementation phase was also assessed for the subgroup of first episodes. A “first episode" referred to a patient not having received chemotherapy in the 6-month period before the start of the episode being assessed.

Secondary outcomes were compared for all the preand post-implementation episodes and for the fully adherent subgroup. In addition, a sensitivity analysis restricted to first episodes compared secondary outcomes for the pre- and post-implementation phases.

Comparisons of dichotomous variables used the chi-square or Fisher exact test; the independent t-test or Mann-Whitney test was used for continuous variables as appropriate. A $p$ value less than 0.05 was considered significant. Statistical analyses were performed using the IBM SPSS Statistics software application (version 24.0 for Windows: IBM, Armonk, NY, U.S.A.).

\section{RESULTS}

\section{Patient Population}

Of 182 episodes consecutively screened for enrolment, $146(80 \%)$ met the eligibility criteria, and 6 were excluded (Figure 1). Overall, 140 episodes involving 102 patients were included. Of those 140 episodes, 70 were in the pre-implementation phase (May 2012 to September 2013). The remaining 70 were in the post-implementation phase (February 2014 to June 2015).

Descriptive characteristics of episodes in the preand post-implementation phases were similar, including the mean duration of study enrolment and of the episode (Table I). The reasons for an episode reaching its end were white cell count recovery $(125 / 140,89.3 \%)$, discharge (10/140, $7.1 \%)$, next chemotherapy cycle (3/140, $2.1 \%)$, and death $(2 / 140,1.4 \%)$, a distribution that was not significantly different between the phases $(p=0.172)$. Antifungal prophylaxis with fluconazole was used in more than $90 \%$ of episodes in each phase. Anti-mold prophylaxis with posaconazole was not prescribed for any episode.

\section{Study Outcomes}

\section{Primary Outcome}

Adherence to the GM screening protocol was observed in 61 of the 70 post-implementation episodes (87\%), with 40 episodes (57\%) being fully adherent and 21 (30\%) being partially adherent. Nonadherence was observed in 9 episodes (13\%) because no GM screens were performed. Full adherence was significantly greater for episodes involving allo-HSCT than for those involving AML or ALL: 11/12 (92\%), $24 / 46(52 \%)$, and 5/12 (42\%) respectively ( $p=0.024)$. For the sub-analysis of first episodes, adherence was observed in

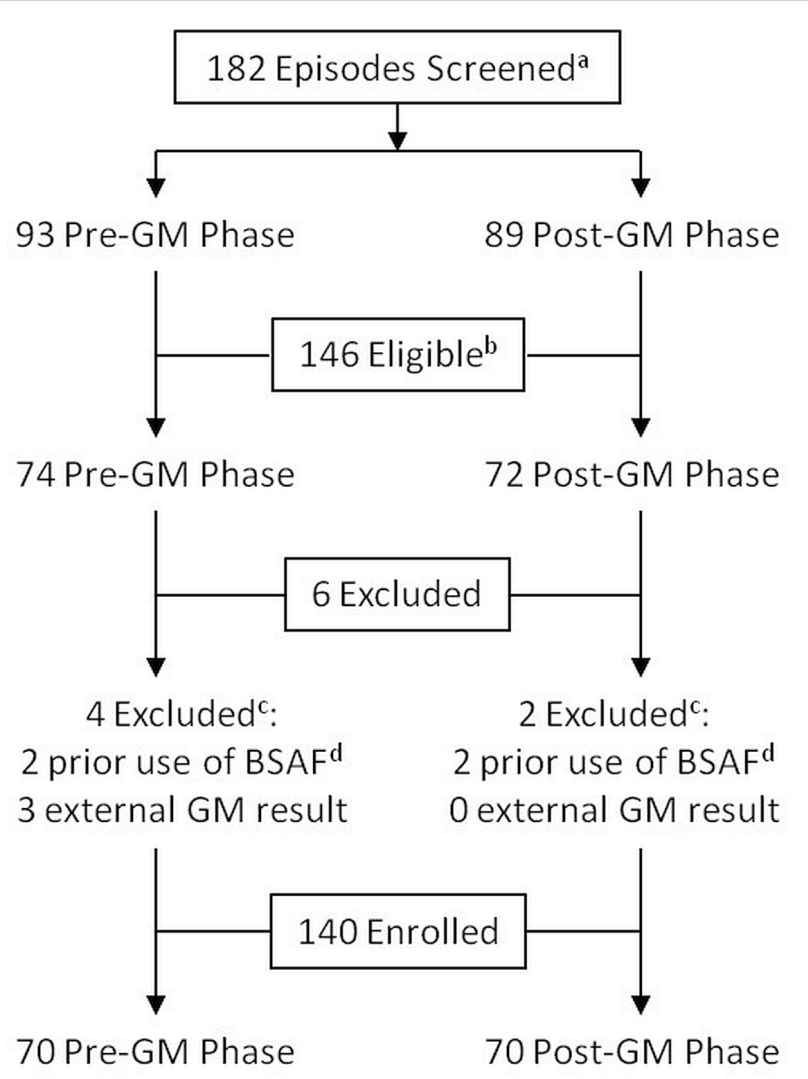

FIGURE 1 Schematic of the study's screening, eligibility, and enrolment process. ${ }^{a}$ Based on chemotherapy protocol. ${ }^{b}$ Based on hematologic diagnosis (matched related allogeneic hematopoietic stem-cell transplantation, or acute myeloid or lymphoid leukemia). 'Individual episodes could have met more than 1 criterion for exclusion. ${ }^{\mathrm{d} B r o a d}$-spectrum antifungal [BSAF (amphotericin B deoxycholate or lipid, caspofungin, or voriconazole)] administered for 3 or more consecutive days during the week before the start of the episode. GM = galactomannan.

43 of 50 episodes ( $86 \%$ ), with 26 episodes (52\%) being fully adherent. Full adherence for first episodes by diagnosis tended to be higher for those involving allo-Hsct (7/8, $87.5 \%)$ than for those involving AML $(16 / 34,47 \%)$ or ALL $(3 / 8,37.5 \%), p=0.080$.

\section{Secondary Outcomes}

Table II shows the data for secondary outcomes. Receipt of BSAFs was significantly more common in preimplementation episodes than in post-implementation episodes ( $27 \%$ vs. $7 \%, p=0.002)$. However, the mean time to BSAF initiation and the mean duration of therapy were similar in both phases. For all episodes receiving BSAFs, voriconazole was most commonly prescribed (16/24), followed by caspofungin $(8 / 24)$. The most common indication for the use of BSAFS was persistent fever (11/19) in the pre-implementation episodes and diagnostic imaging $(3 / 5)$ in the post-implementation episodes. At least 1 complication attributed to BSAFs was documented in 8 of 19 preimplementation episodes and in 1 of 5 post-implementation episodes. Compared with post-implementation episodes, significantly more pre-implementation episodes involved 
TABLE I Characteristics of episodes relative to implementation of the galactomannan screening protocol

\begin{tabular}{|c|c|c|c|c|c|}
\hline \multirow[t]{2}{*}{ Characteristic } & \multirow{2}{*}{$\begin{array}{c}\text { Before } \\
\text { screening }\end{array}$} & \multicolumn{4}{|c|}{ After screening } \\
\hline & & $\begin{array}{c}\text { All } \\
\text { episodes }\end{array}$ & $\begin{array}{c}p \\
\text { value }\end{array}$ & $\begin{array}{l}\text { Full-adherence } \\
\text { episodes }\end{array}$ & $\begin{array}{c}p \\
\text { Value }\end{array}$ \\
\hline Episodes assessed $(n)$ & 70 & 70 & & 40 & \\
\hline Mean age (years) & $56.3 \pm 13.2$ & $55.1 \pm 13.6$ & 0.593 & $54.0 \pm 12.4$ & 0.375 \\
\hline Sex $[n(\%)$ men $]$ & $34(49)$ & $40(57)$ & 0.310 & $20(50)$ & 0.885 \\
\hline Diagnosis $[n(\%)]$ & & & 0.576 & & 0.100 \\
\hline AML & $51(73)$ & $46(66)$ & & $24(60)$ & \\
\hline ALL & $11(16)$ & $12(17)$ & & $5(13)$ & \\
\hline Allo-HSCT & $8(11)$ & $12(17)$ & & $11(28)$ & \\
\hline First episodes ${ }^{\mathrm{a}}[n(\%)]$ & $52(74)$ & $50(71)$ & 0.704 & $26(65)$ & 0.302 \\
\hline \multicolumn{6}{|l|}{ Neutropeniab } \\
\hline Occurrence $[n(\%)]$ & $70(100)$ & $70(100)$ & NA & $40(100)$ & NA \\
\hline Mean duration (days) & $17.9 \pm 8.0$ & $18.3 \pm 8.0$ & 0.750 & $16.5 \pm 7.3$ & 0.360 \\
\hline \multicolumn{6}{|l|}{ Mean duration (days) } \\
\hline Of study enrolment & $57.1 \pm 15.5$ & $59.8 \pm 16.2$ & 0.315 & $58.8 \pm 16.4$ & 0.589 \\
\hline Of episodes & $22.7 \pm 7.6$ & $23.0 \pm 7.9$ & 0.802 & $20.9 \pm 6.5$ & 0.211 \\
\hline Fluconazole prophylaxis [ $n(\%)]$ & $64(91)$ & $67(96)$ & 0.301 & $38(95)$ & 0.488 \\
\hline Broad-spectrum antibiotics ${ }^{\mathrm{C}}[n(\%)]$ & $66(94)$ & $68(97)$ & 0.404 & $39(98)$ & 0.436 \\
\hline
\end{tabular}

Patients not having received chemotherapy for 6 or more months.

b Absolute neutrophil count less than $0.5 \times 10^{9} / \mathrm{L}$, or if neutrophil count not available, total peripheral leucocyte count less than $1 \times 10^{9} / \mathrm{L}$.

c Ceftazidime, ciprofloxacin, imipenem, meropenem, or piperacillin-tazobactam for 3 or more consecutive days.

$\mathrm{AML}=$ acute myeloid leukemia; $\mathrm{ALL}=$ acute lymphoid leukemia; Allo-HSCT = matched related allogeneic hematopoietic stem-cell transplantation; $\mathrm{NA}=$ not applicable.

at least 1 consultation ( $46 \%$ vs. $26 \%, p=0.014$ ), primarily with the Infectious Diseases service. Although more episodes in the pre-implementation phase involved diagnostics (computed tomography imaging or bronchoalveolar lavage), the difference between phases was nonsignificant.

All-cause mortality was available for the 140 enrolled episodes, and it was not significantly different between the phases. However, clinical success at the final assessment was observed in significantly fewer pre-implementation episodes than in post-implementation episodes $(79 \%$ vs. $98 \%, p<0.001)$. Findings in the subgroup of fully adherent post-implementation episodes demonstrated significance similar to that seen in the overall population, with the additional observation that significantly fewer episodes involved diagnostics.

A sensitivity analysis restricted to first episodes showed no significant differences in baseline characteristics. Significantly more pre-implementation episodes involved receipt of BSAFs and consultations (mainly with the Infectious Diseases service), and significantly fewer showed clinical success, but with no difference in all-cause mortality (Tables III and IV).

\section{DISCUSSION}

Adult hematology inpatients with neutropenia at high risk of IFD and receiving fluconazole prophylaxis are the population identified by the Infectious Diseases Society of America as those for whom serial serum GM screening is most likely to be a useful adjunctive test in the diagnosis of aspergillosis ${ }^{14}$. At our centre, a GM screening protocol was implemented to optimize BSAF use. The present study assesses clinician adherence to the GM screening protocol and compares patient outcomes and the use of BSAFs and selected resources before and after implementation of the GM protocol.

Our results show that adherence to the GM screening protocol was observed in most post-implementation episodes (87\%). We also found favourable results for the secondary outcomes studied, including fewer episodes involving receipt of BSAFs, consultations, and diagnostics after implementation of the GM screening protocol. Moreover, despite the decreased use of those resources, significantly more episodes in the post-implementation phase showed clinical success at the final assessment, but with no difference in all-cause mortality.

We observed that full adherence to the protocol was significantly greater for episodes of allo-нsct than for those of induction chemotherapy for acute leukemia. We speculate that the higher adherence rate in allo-нscT episodes is related to those admissions being elective, with their day-to-day management being overseen by a consistent team, which is a situation different than that for the acute leukemia patients.

Prospective trials investigating the incorporation of $\mathrm{GM}$ as the sole non-culture-based marker into a pre-emptive diagnostic-driven strategy have examined the use of BSAFS as a key outcome and have reported reductions ranging 
TABLE II Secondary outcomes for episodes relative to implementation of the galactomannan screening protocol

\begin{tabular}{|c|c|c|c|c|c|}
\hline \multirow[t]{2}{*}{ Outcome } & \multirow{2}{*}{$\begin{array}{c}\text { Before } \\
\text { screening }\end{array}$} & \multicolumn{4}{|c|}{ After screening } \\
\hline & & $\begin{array}{c}\text { All } \\
\text { episodes }\end{array}$ & $\begin{array}{c}p \\
\text { Value }^{\mathrm{a}}\end{array}$ & $\begin{array}{l}\text { Full-adherence } \\
\text { episodes }\end{array}$ & $\begin{array}{c}p \\
\text { Value }^{\mathrm{a}}\end{array}$ \\
\hline Episodes assessed $(n)$ & 70 & 70 & & 40 & \\
\hline \multicolumn{6}{|l|}{ Broad-spectrum antifungals ${ }^{b}$} \\
\hline Used $[n(\%)]$ & $19(27)$ & $5(7)$ & 0.002 & $0(0)$ & $<0.001$ \\
\hline Mean time to start (days) & $15.1 \pm 7.0$ & $14.2 \pm 7.2$ & 0.800 & NA & NA \\
\hline Mean duration of therapy (days) & $10.3 \pm 5.4$ & $9.0 \pm 3.2$ & 0.610 & NA & NA \\
\hline \multicolumn{6}{|l|}{ Consultations during an episode $[n(\%)]$} \\
\hline$\geq 1$ With infectious diseases or respirology & $32(46)$ & $18(26)$ & 0.014 & $8(20)$ & 0.007 \\
\hline With infectious diseases & $30(43)$ & $16(23)$ & 0.012 & $6(15)$ & 0.003 \\
\hline With respirology & $12(17)$ & $6(9)$ & 0.130 & $2(5)$ & 0.066 \\
\hline \multicolumn{6}{|l|}{ Diagnostics during an episode $[n(\%)]$} \\
\hline$\geq 1 \mathrm{CT}$ or $\mathrm{BAL}$ & $32(46)$ & $22(31)$ & 0.083 & $10(25)$ & 0.032 \\
\hline$\geq 1 \mathrm{CT}$ & $30(43)$ & $22(31)$ & 0.162 & $10(25)$ & 0.061 \\
\hline$\geq 1 \mathrm{BAL}$ & $8(11)$ & $3(4)$ & 0.116 & $1(3)$ & 0.151 \\
\hline \multicolumn{6}{|l|}{ Outcomes $[n(\%)]$} \\
\hline All-cause mortality ${ }^{c}$ & $8(11)$ & $4(6)$ & 0.366 & $2(5)$ & 0.322 \\
\hline \multirow[t]{2}{*}{ Clinical success ${ }^{\mathrm{d}}$} & $49(79)$ & $65(98)$ & $<0.001$ & $38(100)$ & 0.002 \\
\hline & (62 episodes) & (66 episodes) & & (38 episodes) & \\
\hline
\end{tabular}

Significant values shown in boldface type.

Amphotericin B deoxycholate or lipid, caspofungin, or voriconazole.

c Death from any cause, assessed at 6 weeks after the end of the episode or at day -1 before subsequent chemotherapy.

d Alive and not taking a broad-spectrum antifungal, assessed at 6 weeks after the end of the episode, or at day -1 before subsequent chemotherapy. $\mathrm{NA}=$ not applicable; $\mathrm{CT}=$ computed tomography; $\mathrm{BAL}=$ bronchoalveolar lavage.

from $11 \%$ to $27 \%^{1,9,13}$. In our study, episodes involving receipt of BSAFs declined by $20 \%$ after implementation of the GM screening protocol. In addition, significantly fewer episodes involved consultations by the Infectious Diseases or Respirology service for infection-related workups. Importantly, those results were sustained in a sensitivity analysis restricted to first episodes (that is, patients who had not received chemotherapy for at least 6 months).

Although our study was not designed to determine the reasons for changes in the use of resources in the postimplementation phase, it is possible that serial negative GM results in a persistently febrile patient who was otherwise clinically well might have increased the clinician's comfort in deferring BSAFS, consultations, or additional diagnostics. The GM test is known to have a high negative predictive value in high-risk hematology patients when disease prevalence is low $^{15}$. The assessment of patient outcomes is of particular importance, given the reductions observed in the use of BSAFs and resources after implementation of the GM screening protocol. Mortality has been assessed at various time points in prospective studies incorporating GM diagnostic testing, with reported rates of approximately $5 \%-18 \%^{1,9,13}$. We chose to assess patient outcomes at 6 weeks after the end of the episode because that period most closely approximates time to mortality from invasive aspergillosis; longer periods might reflect death from confounding causes ${ }^{16,17}$. The all-cause mortality was $2 \%$ for first episodes, which represented individual patients, and $6 \%$ for all episodes after implementation of the GM screening protocol. Our observations of a lack of significant difference in all-cause mortality and significantly more episodes showing clinical success at the final assessment after GM protocol implementation suggests that patients were safely left without treatment. In addition, fewer episodes involved antifungal-related complications.

The limitations of our study include its retrospective design and small sample size (given our available resources). Despite the design, a complete dataset was available for all episodes enrolled. In addition, efforts were made to ensure accuracy by piloting the data collection tool before study initiation and by incorporating an independent data audit for $10 \%$ of episodes. Although the sample size is small, significant differences were observed for the use of BSAFS and consultation services, and for the number of episodes showing clinical success at final assessment. Importantly, the significant differences observed for the overall study population remained consistent for the subgroups of fully adherent post-implementation episodes and first episodes. Although changes in concurrent practices over the study period might have contributed to the favourable differences between phases, the treating hematologists and the chemotherapy protocols used remained consistent over the 36 -month study period. In addition, decisions about the use of BSAFs, consultations, and diagnostics were at the discretion of clinicians in both study phases.

The GM screening protocol in high-risk patients receiving fluconazole prophylaxis has been in use at our centre 
TABLE III Characteristics of first episodes relative to implementation of the galactomannan screening protocol

\begin{tabular}{|c|c|c|c|}
\hline \multirow[t]{2}{*}{ Characteristic } & \multicolumn{2}{|c|}{$\begin{array}{l}\text { Implementation } \\
\text { of screening }\end{array}$} & \multirow[t]{2}{*}{$\begin{array}{c}p \\
\text { Value }\end{array}$} \\
\hline & Before & After & \\
\hline Episodes assessed $(n)$ & 52 & 50 & \\
\hline Mean patient age (years) & $55.5 \pm 14.1$ & $55.0 \pm 14.5$ & 0.882 \\
\hline $\operatorname{Sex}[n(\%)$ men $]$ & $23(44)$ & $28(56)$ & 0.235 \\
\hline Diagnosis $[n(\%)]$ & & & 0.428 \\
\hline AML & $39(75)$ & $34(68)$ & \\
\hline ALL & $9(17)$ & $8(16)$ & \\
\hline Allo-HSCT & $4(8)$ & $8(16)$ & \\
\hline \multicolumn{4}{|l|}{ Neutropenia $^{\mathrm{a}}$} \\
\hline Occurrence $[n(\%)]$ & $52(100)$ & $50(100)$ & NA \\
\hline Mean duration (days) & $18.8 \pm 8.6$ & $19.3 \pm 8.1$ & 0.760 \\
\hline \multicolumn{4}{|l|}{ Mean duration (days) } \\
\hline Of study enrolment & $57.0 \pm 17.2$ & $61.1 \pm 17.0$ & 0.158 \\
\hline Of episodes & $23.6 \pm 8.0$ & $24.3 \pm 8.2$ & 0.688 \\
\hline Fluconazole prophylaxis [n (\%)] & $47(90)$ & $49(98)$ & 0.102 \\
\hline Broad-spectrum antibiotics ${ }^{\mathrm{b}}[n(\%)]$ & $49(94)$ & $48(96)$ & 0.679 \\
\hline
\end{tabular}

a Absolute neutrophil count less than $0.5 \times 10^{9} / \mathrm{L}$, or if neutrophil count not available, total peripheral leucocyte count less than $1 \times 10^{9} / \mathrm{L}$.

b Ceftazidime, ciprofloxacin, imipenem, meropenem, or piperacillin-tazobactam for 3 or more consecutive days.

$\mathrm{AML}=$ acute myeloid leukemia; $\mathrm{ALL}=$ acute lymphoid leukemia; Allo-HSCT $=$ matched related allogeneic hematopoietic stem-cell transplantation; $\mathrm{NA}=$ not applicable.

for nearly 4 years. Our practice is evolving based on continuous monitoring of the local epidemiology of IFD and on changes in patterns of antifungal use for prophylaxis ${ }^{18,19}$.

\section{CONCLUSIONS}

This retrospective cohort study found that adherence to a GM screening protocol was $87 \%$ and was significantly greater for the allo-нscт population than for acute leukemia populations. Implementation of a GM screening protocol in high-risk hematology inpatients with neutropenia and receiving fluconazole prophylaxis was feasible and was associated with significantly fewer episodes involving receipt of BSAFs and consultations and significantly more episodes showing clinical success. Those results support current Infectious Diseases Society of America recommendations ${ }^{14}$.

\section{ACKNOWLEDGMENTS}

The authors express their gratitude to Michael Miller PhD, Department of Pediatrics, Western University, London, ON, for statistical support.

\section{CONFLICT OF INTEREST DISCLOSURES}

We have read and understood Current Oncology's policy on disclosing conflicts of interest, and we declare that we have none.

\section{AUTHOR AFFILIATIONS}

*Canadian Agency for Drugs and Technologies in Health, Toronto, ON; ${ }^{\dagger}$ Pharmacy Services, London Health Sciences Centre, London,
TABLE IV Secondary outcomes for first episodes relative to implementation of the galactomannan screening protocol

\begin{tabular}{|c|c|c|c|}
\hline \multirow[t]{2}{*}{ Outcome } & \multicolumn{2}{|c|}{$\begin{array}{l}\text { Implementation } \\
\text { of screening }\end{array}$} & \multirow[t]{2}{*}{$\underset{\text { Value }}{p}$} \\
\hline & Before & After & \\
\hline Episodes assessed $(n)$ & 52 & 50 & \\
\hline \multicolumn{4}{|l|}{ Broad-spectrum antifungals ${ }^{b}$} \\
\hline Used $[n(\%)]$ & $12(23)$ & $4(8)$ & 0.036 \\
\hline Mean time to start (days) & $17.0 \pm 4.8$ & $15.5 \pm 7.6$ & 0.644 \\
\hline Mean duration of therapy (days) & $9.9 \pm 4.3$ & $10.0 \pm 2.6$ & 0.971 \\
\hline \multicolumn{4}{|l|}{ Consultations during an episode $[n(\%)]$} \\
\hline$\geq 1$ With infectious diseases or respirology & $22(42)$ & $10(20)$ & 0.015 \\
\hline With infectious diseases & $20(38)$ & $9(18)$ & 0.022 \\
\hline With respirology & $9(17)$ & $5(10)$ & 0.284 \\
\hline \multicolumn{4}{|l|}{ Diagnostics during an episode $[n(\%)]$} \\
\hline$\geq 1 \mathrm{CT}$ or $\mathrm{BAL}$ & $21(40)$ & $17(34)$ & 0.505 \\
\hline$\geq 1 \mathrm{CT}$ & $20(38)$ & $17(34)$ & 0.639 \\
\hline$\geq 1 \mathrm{BAL}$ & $6(12)$ & $3(6)$ & 0.488 \\
\hline \multicolumn{4}{|l|}{ Outcomes $[n(\%)]$} \\
\hline All-cause mortality ${ }^{\mathrm{C}}$ & $4(8)$ & $1(2)$ & 0.363 \\
\hline Clinical success ${ }^{\mathrm{d}}$ & $40(83)$ & $48(98)$ & 0.016 \\
\hline & (48 eps) & (49 eps) & \\
\hline
\end{tabular}

Significant values shown in boldface type.

b Amphotericin B deoxycholate or lipid, caspofungin, or voriconazole. Death from any cause, assessed at 6 weeks after the end of the episode or at day -1 before subsequent chemotherapy.

d Alive and not taking a broad-spectrum antifungal, assessed at 6 weeks after the end of the episode or at day -1 before subsequent chemotherapy.

$\mathrm{CT}=$ computed tomography; $\mathrm{BAL}=$ bronchoalveolar lavage; eps = episodes.

ON; ${ }^{\ddagger}$ Department of Medicine, Schulich School of Medicine and Dentistry, Western University, London, ON; ${ }^{\S}$ Department of Medicine, Division of Hematology, Schulich School of Medicine and Dentistry, Western University, London, ON; "Department of Epidemiology and Biostatistics, Schulich School of Medicine and Dentistry, Western University, London, ON; ${ }^{\#}$ Department of Medicine, Division of Infectious Diseases, Schulich School of Medicine and Dentistry, Western University, London, ON; **Department of Pathology and Laboratory Medicine, Schulich School of Medicine and Dentistry, Western University, London, $\mathrm{ON} ;{ }^{\dagger \dagger}$ Arthur Labatt Family School of Nursing, Western University, London, ON; ${ }^{\text {执De- }}$ partment of Microbiology and Immunology, Schulich School of Medicine and Dentistry, Western University, London, ON.

\section{REFERENCES}

1. Maertens J, Theunissen K, Verhoef G, et al. Galactomannan and computed tomography-based preemptive antifungal therapy in neutropenic patients at high risk for invasive fungal infection: a prospective feasibility study. Clin Infect Dis 2005;41:1242-50.

2. Freifeld AG, Bow EJ, Sepkowitz KA, et al. on behalf of the Infectious Diseases Society of America. Clinical practice guideline for the use of antimicrobial agents in neutropenic patients with cancer: 2010 update by the Infectious Diseases Society of America. Clin Infect Dis 2011;52:e56-93. 
3. Morrissey CO, Gilroy NM, Macesic N, et al. Consensus guidelines for the use of empiric and diagnostic-driven antifungal treatment strategies in haematological malignancy, 2014. Intern Med J 2014;44:1298-314.

4. Maertens JA, Nucci M, Donnelly JP. The role of antifungal treatment in hematology. Haematologica 2012;97:325-7.

5. Girmenia C, Aversa F, Busca A, et al. on behalf of the Sorveglianza Epidemiologica delle Infezioni Fungine nelle Emopatie Maligne, the Invasive Fungal Infections Cooperative Group of the European Organization for Research and Treatment of Cancer, the Gruppo Italiano Malattie Ematologiche dell'Adulto, the Associazione Italiana Ematologia ed Oncologia Pediatrica, and the Gruppo Italiano Trapianto di Midollo Osseo. A hematology consensus agreement on antifungal strategies for neutropenic patients with hematological malignancies and stem cell transplant recipients. Hematol Oncol 2013;31:117-26.

6. Cordonnier C, Robin C, Alanio A, Bretagne S. Antifungal pre-emptive strategy for high-risk neutropenic patients: why the story is still ongoing. Clin Microbiol Infect 2014;20(suppl 6):27-35.

7. Herbrecht R, Berceanu A. Beta-D-glucan detection test: a step toward preemptive therapy for fungal infections in leukemic patients? Clin Infect Dis 2008;46:886-9.

8. de Pauw BE. Between over- and undertreatment of invasive fungal disease. Clin Infect Dis 2005;41:1251-3.

9. Tan BH, Low JG, Chlebicka NL, et al. Galactomannan-guided preemptive vs. empirical antifungals in the persistently febrile neutropenic patient: a prospective randomized study. Int J Infect Dis 2011;15:e350-6.

10. FungM,KimJ,MartyFM,SchwarzingerM,KooS. Meta-analysis and cost comparison of empirical versus pre-emptive antifungal strategies in hematologic malignancy patients with high-risk febrile neutropenia. PLoS One 2015;10:e0140930.
11. Miceli MH, Maertens J. Role of non-culture-based tests, with an emphasis on galactomannan testing for the diagnosis of invasive aspergillosis. Semin Respir Crit Care Med 2015;36:650-61.

12. de Pauw B, Walsh TJ, Donnelly JP, et al. Revised definitions of invasive fungal disease from the European Organization for Research and Treatment of Cancer/Invasive Fungal Infections Cooperative Group and the National Institute of Allergy and Infectious Diseases Mycoses Study Group (EORTC/MSG) Consensus Group. Clin Infect Dis 2008;46:1813-21.

13. Cordonnier C, Pautas C, Maury S, et al. Empirical versus preemptive antifungal therapy for high-risk, febrile, neutropenic patients: a randomized, controlled trial. Clin Infect Dis 2009;48:1042-51.

14. Patterson TF, Thompson GR 3rd, Denning DW, et al. Practice guidelines for the diagnosis and management of aspergillosis: 2016 update by the Infectious Diseases Society of America. Clin Infect Dis 2016;63:e1-60.

15. Pfeiffer CD, Fine JP, Safdar N. Diagnosis of invasive aspergillosis using a galactomannan assay: a meta-analysis. Clin Infect Dis 2006;42:1417-27.

16. Wingard JR, Ribaud P, Schlamm HT, Herbrecht R. Changes in causes of death over time after treatment for invasive aspergillosis. Cancer 2008;112:2309-12.

17. Marr KA, Schlamm HT, Herbrecht R, et al. Combination antifungal therapy for invasive aspergillosis: a randomized trial. Ann Intern Med 2015;162:81-9. [Erratum in: Ann Intern Med 2015;162:463]

18. Omrani AS, Almaghrabi RS. Complications of hematopoietic stem transplantation: fungal infections. Hematol Oncol Stem Cell Ther 2017;10:239-44.

19. Macesic N, Morrissey CO, Liew D, et al. Is a biomarker-based diagnostic strategy for invasive aspergillosis cost effective in high-risk haematology patients? Med Mycol 2017;55:705-12. 